\title{
Ueber die Determinante, deren Elemente die Quadrate der sechszehn Verbindungslinien der Eckpunkte zweier beliebiger Tetraeder sind.
}

(Von Herrn Siebeck zu Liegnitz.)

I.
Das Product der Volumina zweier beliebiger Tetraeder $P$ und $\Pi$ kann bekanntlich als rationale Function der Quadrate der sechszehn Verbindungslinien der Eckpunkte der beiden Tetraeder und zwar in Determinantenform ausgedrückt werden. Sind nämlich $E_{1} E_{2} E_{3} E_{4}$ und $\mathrm{E}_{1} \mathrm{E}_{2} \mathrm{E}_{3} \mathrm{E}_{4}$ die Ecken von $\boldsymbol{P}$ und $\boldsymbol{I I}$ und bezeichnet man das Quadrat der Strecke $\boldsymbol{E}_{i} \mathbf{E}_{k}$. durch $e_{i k}$ so ist, wie bekannt,

$$
\text { (1.) }\left|\begin{array}{lllll}
0 & 1 & 1 & 1 & 1 \\
1 & e_{11} & e_{12} & e_{13} & e_{14} \\
1 & e_{21} & e_{22} & e_{23} & e_{24} \\
1 & e_{31} & e_{32} & e_{33} & e_{34} \\
1 & e_{41} & e_{42} & e_{43} & e_{44}
\end{array}\right|=288 E_{1} E_{2} E_{3} E_{4} \cdot \mathrm{E}_{1} \mathrm{E}_{2} \mathrm{E}_{3} \mathrm{E}_{4}
$$

Aus dieser Gleichung leitet man, indem man beide Tetraeder congruiren lässt, u. A. unmittelbar die Gleichung her, welche das Volumen eines Tetraeders durch seine sechs Kanten ausdrückt. Der betreffenden Formel steht aber bekanntlich eine andere von Crelle (math. Aufs.), Joachimsthal (Bd. 40 d. J.), v. Staudt (Bd. 47 d. J.) behandelte gegenüber, mittelst welcher das Product $P \boldsymbol{r}$ (wo $r$ der Halbmesser der dem $\boldsymbol{P}$ umschriebenen Kugel) durch die sechs Kanten des Tetraeders ausgedrückt wird. Zu dieser letzteren Formel lässt sich aber, wie ich sogleich zeigen werde, ebenfalls eine allgemeinere finden, vermöge welcher, wenn $\varrho$ der Radius der dem $\Pi$ umschriebenen Kugel ist, das Product Pr. $\Pi_{\varphi}$ (in ähnlicher Weise, wie das Product $P \Pi$ in obiger Gleichung) durch eine aus den Quadraten jener sechszehn Linien gebildete Determinante und durch den Winkel, unter welchem sich die beiden Kugeln schneiden, in einfachster Weise ausgedrückt werden kann. Nennen wir nämlich diesen Winkel $\varphi$, so behaupten wir, dass 


$$
\left|\begin{array}{llll}
e_{11} & e_{12} & e_{13} & e_{14} \\
e_{21} & e_{22} & e_{23} & e_{24} \\
e_{31} & e_{32} & e_{33} & e_{34} \\
e_{41} & e_{42} & e_{43} & e_{44}
\end{array}\right|=576 \operatorname{Pr} \cdot \Pi \varrho \cdot \cos \varphi
$$

Nehmen wir, um dies zu beweisen, ein rechtwinkliges Coordinatensystem an und nennen $x_{i}, y_{i}, z_{i}$ die Coordinaten von $E_{i} ; \xi_{i}, \eta_{i}, \zeta_{i}$ die Coordinaten von $\mathrm{E}_{i}$, seien endlich $a, b, c$ und $\alpha, \beta, \gamma$ die Coordinaten der Mittelpunkte $C$ und $\boldsymbol{\Gamma}$ der dem $\boldsymbol{P}$ und $\Pi$ umschriebenen Kugeln, so erhalten wir zunächst für das Quadrat der Centralen $C I$ die Gleichung

$$
\text { (3.) } \quad(a-\alpha)^{2}+(b-\beta)^{2}+(c-\gamma)^{2}=r^{2}+\rho^{2}+2 r \rho \cos \varphi \text {. }
$$

Ferner ist

$$
\begin{array}{ll}
\text { (4.) } & \left(x_{i}-\xi_{k}\right)^{2}+\left(y_{i}-\eta_{k}\right)^{2}+\left(\boldsymbol{z}_{i}-\zeta_{k}\right)^{2}=e_{i k}, \\
\text { (5.) } \quad & \left(x_{i}-a\right)^{2}+\left(y_{i}-b\right)^{2}+\left(z_{i}-c\right)^{2}=r^{2}, \\
\left(5^{*} .\right) & \left(\xi_{k}-\alpha\right)^{2}+\left(\eta_{k}-\beta\right)^{2}+\left(\zeta_{k}-\gamma\right)^{2}=\varrho^{2} .
\end{array}
$$

Durch Addition von (3.) und (4.), ferner von (5.) und $\left(5^{*}\right.$.), und nachherige Subtraction erhält man

$$
\text { (6.) }\left(x_{i}-\alpha\right)\left(\xi_{k}-a\right)+\left(y_{i}-\beta\right)\left(\eta_{k}-b\right)+\left(z_{i}-\gamma\right)\left(\zeta_{k}-c\right)+r \varrho \cos \varphi=-\frac{1}{2} e_{i k} \text {. }
$$

Letztere Gleichung bildet das Schema für sechszehn Gleichungen, welche man erhält, wenn man den Indices allmählig sämmtliche Werthe von 1 bis 4 ertheilt. Aus diesen sechszehn Gleichungen ergiebt sich aber unter Benutzung des Multiplicationstheorems für Determinanten, die nachfolgende

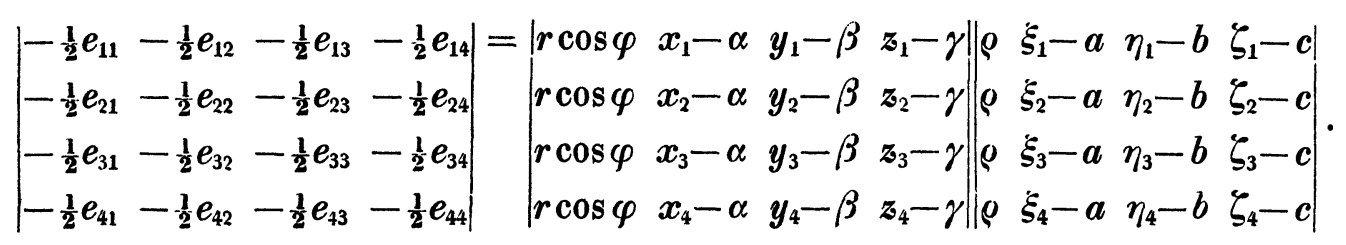
Somit ist

$$
\stackrel{\frac{1}{16}}{e_{11}} \begin{array}{llll}
e_{12} & e_{13} & e_{14} \\
e_{21} & e_{22} & e_{23} & e_{24} \\
e_{31} & e_{32} & e_{33} & e_{34} \\
e_{41} & e_{42} & e_{43} & e_{44}
\end{array}|=| \begin{array}{llll|llll}
1 & x_{1} & y_{1} & z_{1} & 1 & \xi_{1} & \eta_{1} & \zeta_{1} \\
1 & x_{2} & y_{2} & z_{2} & 1 & \xi_{2} & \eta_{2} & \zeta_{2} \\
1 & x_{3} & y_{3} & z_{3} & 1 & \xi_{3} & \eta_{3} & \zeta_{3} \\
1 & x_{4} & y_{4} & z_{4} & 1 & \xi_{4} & \eta_{4} & \zeta_{4}
\end{array} \mid
$$

Aus dieser Gleichung ergiebt sich aber ohne Weiteres die Gleichung (2.); und zwar erhalten wir aus der letzteren durch Benutzung von (1.) zugleich die nachfolgende 


$$
\text { (7.) }\left|\begin{array}{rrrrr}
-\frac{1}{2 r \varrho \cos \varphi} & 1 & 1 & 1 & 1 \\
1 & e_{11} & e_{12} & e_{13} & e_{14} \\
1 & e_{21} & e_{22} & e_{23} & e_{24} \\
1 & e_{31} & e_{32}^{*} & e_{33} & e_{34} \\
1 & e_{41} & e_{42} & e_{43} & e_{44}
\end{array}\right|=0
$$

\section{II.}

Die Gleichung (2.) giebt zunächst folgenden bemerkenswerthen Satz: Drehen sich zwei Tetraeder auf beliebige Weise um die als unbeweglich gedachten Mittelpunkte der ihnen umschriebenen Kugeln, so behält die Determinante

$$
\left|\begin{array}{llll}
e_{11} & e_{12} & e_{13} & e_{14} \\
e_{21} & e_{22} & e_{23} & e_{24} \\
e_{31} & e_{32} & e_{33} & e_{34} \\
e_{41} & e_{42} & e_{43} & e_{44}
\end{array}\right|
$$

beständig denselben Werth.

Lassen wir ferner beide Tetraeder congruiren, indem wir $e_{i k}=e_{k i}$, $e_{i i}=0, \cos \varphi=-1$ setzen, so erhalten wir aus (2.) die schon oben erwähnte bekannte Gleichung

$$
\text { (8.) }\left|\begin{array}{llll}
0 & e_{12} & e_{13} & e_{14} \\
e_{12} & 0 & e_{23} & e_{24} \\
e_{13} & e_{23} & 0 & e_{34} \\
e_{14} & e_{24} & e_{34} & 0
\end{array}\right|=-576 P^{2} r^{2}
$$

in welcher sich bekanntlich die Determinante in vier Factoren zerlegen lässt. Ebenso giebt auch die Formel (7.) einen Ausdruck für den Radius der dem $P$ umschriebenen Kugel.

Setzen wir in (2.) $\cos \varphi=+1$, so erhalten wir die Bedingungsgleichung für die äussere Berührung, setzen wir $\cos \varphi=-1$, die für die innere Berührung der beiden Kugeln. Durch Verbindung von (2.) mit (8.) erhält man so eine Bedingungsgleichung, welche sämmtliche acht und zwanzig Verbindungslinien der acht Punkte $E_{1} E_{2} E_{3} E_{4}, E_{1} E_{2} E_{3} E_{4}$ enthält. Bezeichnen wir nämlich durch $\partial_{i k}$ das Quadrat von $E_{i} E_{k}$, und durch $\partial_{i k}^{\prime}$ das Quadrat von $E_{i} E_{k}$, so heisst diese Gleichung 


$$
\left|\begin{array}{llll}
e_{11} & e_{12} & e_{13} & e_{14} \\
e_{21} & e_{22} & e_{23} & e_{24} \\
e_{31} & e_{32} & e_{33} & e_{34} \\
e_{41} & e_{42} & e_{43} & e_{44}
\end{array}\right|-\left|\begin{array}{llll}
0 & \partial_{12} & \partial_{13} & \partial_{14} \\
\partial_{12} & 0 & \partial_{23} & \partial_{24} \\
\partial_{13} & \partial_{23} & 0 & \partial_{34} \\
\partial_{14} & \partial_{24} & \partial_{34} & 0
\end{array}\right|\left|\begin{array}{llll}
0 & \partial_{12}^{\prime} & \partial_{13}^{\prime} & \partial_{14}^{\prime} \\
\partial_{12}^{\prime} & 0 & \partial_{23}^{\prime} & \partial_{24}^{\prime} \\
\partial_{13}^{\prime} & \partial_{23}^{\prime} & 0 & \partial_{34}^{\prime} \\
\partial_{14}^{\prime} & \partial_{24}^{\prime} & \partial_{34}^{\prime} & 0
\end{array}\right|=0 .
$$

Lassen wir in (2.) $E_{1}$ mit $E_{1}, E_{2}$ mit $E_{2}, E_{3}$ mit $E_{3}$, nicht aber $E_{4}$ mit $E_{4}$ zusammenfallen und setzen $\cos \varphi=-1$, so erhalten wir in Verbindung mit (8.) eine Bedingungsgleichung dafür, dass die fünf Punkte $E_{1} E_{2} E_{3} E_{4} E_{4}$ auf der Oberfläche einer Kugel liegen. Diese Gleichung enthält nur die zehn Verbindungslinien jener fünf Punkte und muss daher mit der von Cayley für diesen Fall gefundenen Bedingungsgleichung dem Wesen nach übereinstimmen, obwohl sie der äusseren Form nach von ihr verschieden ist.

\section{1 .}

Als der bemerkenswertheste unter den besonderen Fällen, welche die Gleichung (2.) umfasst, erscheint derjenige, in welchem $\varphi=\frac{1}{2} \pi$. Wir erhalten dann den Satz: Sind die beiden den Tetraedern $P$ und $\Pi$ umschriebenen Kugeln orthogonal, so ist die Determinante

$$
\left|\begin{array}{llll}
e_{11} & e_{12} & e_{13} & e_{14} \\
e_{21} & e_{22} & e_{23} & e_{24} \\
e_{31} & e_{32} & e_{33} & e_{34} \\
e_{41} & e_{42} & e_{43} & e_{44}
\end{array}\right|=0
$$

und umgekehrt: ist diese Determinante $=0$, so sind die beiden Kugeln orthogonal. Das letztere folgt daraus, dass in dem Product $\operatorname{Pr} . \Pi \varrho . \cos \varphi$ einer der beiden Factoren $\operatorname{Pr}$ und $\Pi_{\emptyset}$ nur dann gleich Null sein kann, wenn die Ecken des betreffenden Tetraeders in einem Kreise liegen, (denn liegen die vier Ecken in einer Ebene und nicht in einem Kreise, so ist zwar das betreffende Tetraeder gleich Null, dagegen der Radius der Kugel unendlich gross, das Product $P r$ aber hat dann immer noch einen endlichen Werth). Liegen aber die vier Ecken in einem Kreise, so lässt sich durch letzteren doch stets eine Kugel legen, welche der anderen orthogonal ist.

Bezeichnen wir nun den Coefficienten von $e_{i k}$ in der Entwickelung der Determinante $\left|\begin{array}{llll}e_{11} & e_{12} & e_{13} & e_{14} \\ e_{12} & e_{2} & e_{3} & e_{24}\end{array}\right|$ durch $\varepsilon_{i k}$, so ist beispielsweise

$$
\left|\begin{array}{llll}
e_{21} & e_{22} & e_{23} & e_{24} \\
e_{31} & e_{32} & e_{33} & e_{34} \\
e_{41} & e_{42} & e_{43} & e_{44}
\end{array}\right|
$$




$$
\varepsilon_{12}=-\left|\begin{array}{lll}
e_{21} & e_{23} & e_{24} \\
e_{31} & e_{33} & e_{34} \\
e_{41} & e_{43} & e_{44}
\end{array}\right|
$$

folglich mit Berücksichtigung von (6.) (wenn man dort $\cos \varphi=0$ setzt) und mit Anwendung des Multiplicationsgesetzes für Determinanten

$$
\frac{1}{8} \varepsilon_{12}=\left|\begin{array}{lll}
x_{2}-\alpha & y_{2}-\beta & z_{2}-\gamma \\
x_{3}-\alpha & y_{3}-\beta & z_{3}-\gamma \\
x_{4}-\alpha & y_{4}-\beta & z_{3}-\gamma
\end{array}\right|\left|\begin{array}{ccc}
\xi_{1}-a & \eta_{1}-b & \zeta_{1}-c \\
\xi_{3}-a & \eta_{3}-b & \zeta_{3}-c \\
\xi_{4}-a & \eta_{4}-b & \zeta_{4}-c
\end{array}\right|=\left|\begin{array}{cccc}
1 & \alpha & \beta & \gamma \\
1 & x_{2} & y_{2} & z_{3} \\
1 & x_{3} & y_{3} & z_{3} \\
1 & x_{4} & y_{4} & z_{4}
\end{array}\right|\left|\begin{array}{cccc}
1 & a & b & c \\
1 & \xi_{1} & \eta_{1} & \zeta_{1} \\
1 & \xi_{3} & \eta_{3} & \zeta_{3} \\
1 & \xi_{4} & \eta_{4} & \zeta_{4}
\end{array}\right| .
$$

Demnach ist

$$
\varepsilon_{12}=288 T^{\prime} E_{2} E_{3} E_{4} \cdot C \mathrm{E}_{1} \mathrm{E}_{3} \mathrm{E}_{4}=288 T E_{2} E_{3} E_{4} . C \mathrm{E}_{3} \mathrm{E}_{4} \mathrm{E}_{1} .
$$

Eben solche Ausdrücke erhält man für die übrigen $\varepsilon$ und zwar ist

(10.)

$$
\left\{\begin{array}{l}
-\varepsilon_{11}=288 I E_{2} E_{3} E_{4} \cdot C \mathrm{E}_{2} \mathrm{E}_{3} \mathrm{E}_{4},-\varepsilon_{21}=288 E_{1} I E_{3} E_{4} \cdot C \mathrm{E}_{2} \mathrm{E}_{3} \mathrm{E}_{4}, \\
-\varepsilon_{12}=288 \Gamma E_{2} E_{3} E_{4} \cdot \mathrm{E}_{1} C \mathrm{E}_{3} \mathrm{E}_{4},-\varepsilon_{22}=288 E_{1} T E_{3} E_{4} \cdot \mathrm{E}_{1} C \mathrm{E}_{3} \mathrm{E}_{4}, \\
-\varepsilon_{13}=288 I E_{2} E_{3} E_{4} \cdot \mathrm{E}_{1} \mathrm{E}_{2} C \mathrm{E}_{4},-\varepsilon_{23}=288 E_{1} I E_{3} E_{4} \cdot \mathrm{E}_{1} \mathrm{E}_{2} C \mathrm{E}_{4}, \\
-\varepsilon_{14}=288 \Gamma E_{2} E_{3} E_{4} \cdot \mathrm{E}_{1} \mathrm{E}_{2} \mathrm{E}_{3} C,-\varepsilon_{24}=288 E_{1} \Gamma E_{3} E_{4} \cdot \mathrm{E}_{1} \mathrm{E}_{2} \mathrm{E}_{3} C
\end{array}\right. \text { etc. }
$$

übereinstimmend mit dem bekannten Satze, wonach, wenn die Determinante verschwindet, sein muss

$$
\varepsilon_{11}: \varepsilon_{12}: \varepsilon_{13}: \varepsilon_{14}=\varepsilon_{21}: \varepsilon_{22}: \varepsilon_{23}: \varepsilon_{24}=\text { etc. }
$$

Vorstehendes kann als Grundlage für die Untersuchung desjenigen Coordinatensystems dienen, in welchem, wenn $x_{1}, x_{2}, x_{3}, x_{4}$ die Quadrate der Entfernungen eines beliebigen Punktes E im Raume von den vier festen Punkten $E_{1}, E_{2}, E_{3}, E_{4}$ sind, die Gleichung einer beliebigen Fläche durch eine homogene Function der $x$ ausgedrückt wird. Nach einem bekannten Satze (von Bodenmiller, bewiesen u. A. von Möbius, Schlömilch und Chasles) giebt es in einer Ebene immer zwei (reelle oder imaginäre) Punkte von der Beschaffenheit, dass ihre Entfernungen von drei festen Punkten derselben Ebene sich wie drei beliebige gegebene Zahlen verhalten, und zwar liegen diese beiden Punkte mit dem Mittelpunkte des durch jene drei festen Punkte gehenden Kreises in gerader Linie und der eine derselben ist der Pol des anderen (im Plïckerschen Sinne). Ein entsprechender Satz gilt auch für den Raum, nämlich für vier feste Punkte $E_{1}, E_{2}, E_{3}, E_{4}$ und die durch sie gelegte Kugel, und es würden sonach bestimmten Werthen von $x_{1}, x_{2}, x_{3}, x_{4}$ nach obiger Coordinatenbestimmung im Allgemeinen immer zwei Punkte, ein ausserhalb 
und ein innerhalb liegender, entsprechen, von denen der eine aus dem anderen immer leicht bestimmt werden kann.

Dies vorausgeschickt, so folgt aus dem Obigen, dass durch eine homogene Gleichung des ersten Grades, etwa

$$
A_{1} x_{1}+A_{2} x_{2}+A_{3} x_{3}+A_{4} x_{4}=0
$$

in obigem Coordinatensystem eine Kugel vorgestellt wird, welche der durch $E_{1} E_{2} E_{3} E_{4}$ gehenden Kugel orthogonal ist. Aus den oben für die $\varepsilon$ gefundenen Werthen folgt nun, dass die Constanten $A_{1}, A_{2}, A_{3}, A_{4}$ in demselben Verhältnisse stehen, wie die Tetraeder $\Gamma E_{2} E_{3} E_{4}, \Gamma E_{3} E_{4} E_{1}, T E_{4} E_{1} E_{2}, I E_{1} E_{2} E_{3}$ und somit diese Constanten weiter nichts sind, als die Coordinaten des Mittelpunktes der orthogonalen Kügel nach dem gewöhnlichen Coordinatensystem. Eine allgemeine Untersuchung, insbesondere derjenigen Oberflächen, welche nach diesem Coordinatensystem durch eine homogene Function zweiten Grades vorgestellt werden, dürfte um so interessantere Resultate versprechen, als aus dem Obigen hervorgeht, dass, wenn wir auch hier den Dualismus in Betreff der Oberflächen zweiter Ordnung und zweiter Klasse zur Geltung bringen, die Oberflächen zweiter Klasse nach unserm Coordinatensystem mit den Oberflächen zweiter Ordnung nach dem gewöhnlichen System übereinstimmen würden.

Schliesslich bleibe nicht unerwähnt, dass, wenn wir $\mathbf{E}_{4}$ zum Mittelpunkt der durch $E_{1} E_{2} E_{3} E_{4}$ gehenden Kugel machen, so dass also die durch $\mathrm{E}_{1} \mathrm{E}_{2} \mathrm{E}_{3} \mathrm{E}_{4}$ gehende der anderen orthogonale Kugel in die Ebene eines grössten Kreises ausartet, die Bedingungsgleichung

in folgende

$$
\left|\begin{array}{llll}
e_{11} & e_{12} & e_{13} & e_{14} \\
e_{21} & e_{22} & e_{23} & e_{24} \\
e_{31} & e_{32} & e_{33} & e_{34} \\
e_{41} & e_{42} & e_{43} & e_{44}
\end{array}\right|=0
$$

$$
\text { (11.) }\left|\begin{array}{llll}
1 & e_{11} & e_{12} & e_{13} \\
1 & e_{21} & e_{22} & e_{23} \\
1 & e_{31} & e_{32} & e_{33} \\
1 & e_{41} & e_{42} & e_{43}
\end{array}\right|=0
$$

übergeht. Letzteres ist also die Bedingung dafür, dass die Punkte $\mathrm{E}_{1} \mathrm{E}_{2} \mathrm{E}_{3}$ in der Ebene eines grössten Kreises der durch $E_{1} E_{2} E_{3} E_{4}$ gelegten Kugel liegen. 
IV.

Aus der Gleichung (2.) lassen sich auch interessante Relationen, welche das Product der sinus zweier Raumwinkel und die ihnen umschriebenen normalen Kegel betreffen, ableiten. Um auch hier wieder das Hauptresultat voranstellen zu können, wollen wir durch $E_{1} E_{2} E_{3}$ und $E_{1} E_{2} E_{3}$ die beiden dreiseitigen Ecken bezeichnen, deren Kanten einen beliebigen Punkt $O$ des Raumes mit $E_{1} E_{2} E_{3}, \mathrm{E}_{1} \mathrm{E}_{2} \mathrm{E}_{3}$ verbinden; ferner werde der Winkel $E_{i} O \mathrm{E}_{k}$ durch $\boldsymbol{e}_{i k}$ bezeichnet, und durch $r^{\prime}$ und $\varrho^{\prime}$ die Winkel, welche die Achsen der jenen beiden Ecken umschriebenen geraden Kegel mit den Seiten der letzteren bilden. Wir behaupten dann, dass analog der Gleichung (2.)

$$
\left|\begin{array}{cccc}
1 & 1 & 1 & 1 \\
1 & \cos e_{11} & \cos e_{12} & \cos e_{13} \\
1 & \cos e_{21} & \cos e_{22} & \cos e_{23} \\
1 & \cos e_{31} & \cos e_{32} & \cos e_{33}
\end{array}\right|=\sin E_{1} E_{2} E_{3} \operatorname{tang} r^{\prime} \cdot \sin E_{1} E_{2} E_{3} \operatorname{tang} \varrho^{\prime} \cdot \cos \psi,
$$

wo $\psi$ der Winkel ist, unter welchem sich die beiden Kegel schneiden.

Lassen wir nämlich in der Gleichung (2.) die Punkte $\boldsymbol{E}_{4}$ und $\mathbf{E}_{4}$ mit $O$ zusammenfallen und sei $O$ der Mittelpunkt einer Kugel, deren Radius $=1$. und auf deren Fläche sich die Punkte $E_{1} E_{2} E_{3} E_{1} E_{2} E_{3}$ befinden, so verwandelt sich die auf der linken Seite der Gleichung (2.) stehende Determinante, wenn man in derselben die letzte Horizontal - und Verticalreihe zur ersten Horizontalund Verticalreihe macht, in

$$
\left|\begin{array}{cccc}
0 & 1 & 1 & 1 \\
1 & 4 \sin ^{2} \frac{1}{2} e_{11} & 4 \sin ^{2} \frac{1}{2} e_{12} & 4 \sin ^{2} \frac{1}{2} e_{13} \\
1 & 4 \sin ^{2} \frac{1}{2} e_{21} & 4 \sin ^{2} \frac{1}{2} e_{22} & 4 \sin ^{2} \frac{1}{2} e_{23} \\
1 & 4 \sin ^{2} \frac{1}{2} e_{31} & 4 \sin ^{2} \frac{1}{2} e_{32} & 4 \sin ^{2} \frac{1}{2} e_{33}
\end{array}\right| .
$$

welche durch eine leichte Reduction auf die Form

$$
\left|\begin{array}{cccc}
0 & 1 & 1 & 1 \\
1 & \cos e_{11} & \cos e_{12} & \cos e_{13} \\
1 & \cos e_{21} & \cos e_{22} & \cos e_{23} \\
1 & \cos e_{31} & \cos e_{32} & \cos e_{33}
\end{array}\right|
$$

gebracht wird. Die Gleichung (2.) hat somit schon folgende Form angenommen:

$$
\text { (13.) }\left|\begin{array}{cccc}
0 & 1 & 1 & 1 \\
1 & \cos e_{11} & \cos e_{12} & \cos e_{13} \\
1 & \cos e_{21} & \cos e_{22} & \cos e_{23} \\
1 & \cos e_{31} & \cos e_{32} & \cos e_{33}
\end{array}\right|=144 \operatorname{Pr} \cdot \Pi \varrho \cdot \cos \varphi \text {, }
$$


und es bleibt nur noch übrig, den auf der rechten Seite stehenden Ausdruck umzuformen. Denke man sich daher durch $O E_{1} E_{2} E_{3}$ und $O E_{1} E_{2} E_{3}$ normale Kegel mit der Spitze $O$ gelegt, und bezeichne die Winkel, welche die Achsen $O B$ und $O B$ derselben mit den Seiten bilden, durch $r^{\prime}$ und $\varrho^{\prime}$; sei ferner $O F$ eine der beiden Durchschnittslinien der Kegel, so ist zunächst, da die Mittelpunkte $C$ und $T$ der beiden durch $O E_{1} E_{2} E_{3}$ und $O E_{1} E_{2} E_{3}$ gehenden Kugeln in $O B$ und $O B$ liegen, der Winkel $C O I^{\prime}=180^{\prime \prime}-\varphi$, und folglich

$$
\text { (14.) }-\cos \varphi=\cos r^{\prime} \cos \varrho^{\prime}-\sin r^{\prime} \sin \varrho^{\prime} \cos \psi \text {. }
$$

Verbindet man nun $C$ und $I$ mit der Mitte $M$ von $O F$, so sind $C O M$ und $I^{\prime} O M$ rechtwinklige Dreiecke, und es ist somit, da $C O=r, I ' O=\varrho, O M=\frac{1}{2}$ ist,

$$
\text { (15.) } r=\frac{1}{2 \cos r^{\prime}}, \quad \varrho=\frac{1}{2 \cos \varrho^{\prime}} \text {. }
$$

Aus (14.) und (15.) folgt somit, dass

$$
\text { (16.) } \quad 4 \text { r } \varrho \cos \varphi=-1+\operatorname{tang} r^{\prime} \text {. tang } \varrho^{\prime} \cos \psi \text {. }
$$

Ausserdem aber ist $P \Pi=36 \sin E_{1} E_{2} E_{3} \sin E_{1} E_{2} E_{3}$, welche Gleichung mit (16.) und (13.) zusammengestellt, die Gleichung (12.) giebt.

Die letztere wäre somit bewiesen, und es lässt sich auch aus ihr, gerade so wie (7.) aus (2.), eine Gleichung herleiten, in welcher die Sinus der Raumwinkel nicht mehr vorkommen. . Nach $v$. Staudt ist nämlich

$$
\left|\begin{array}{ccc}
\cos \mathfrak{e}_{11} & \cos \mathrm{e}_{12} & \cos \mathrm{e}_{13} \\
\cos \mathrm{e}_{21} & \cos \mathrm{e}_{22} & \cos \mathrm{e}_{23} \\
\cos \mathrm{e}_{31} & \cos \mathrm{e}_{32} & \cos \mathrm{e}_{33}
\end{array}\right|=\sin E_{1} E_{2} E_{3} \cdot \sin \mathrm{E}_{1} \mathrm{E}_{2} \mathrm{E}_{3} .
$$

Dies in Verbindung mit (12.) giebt

$$
\left|\begin{array}{cccc}
1-\operatorname{tang} r^{\prime} \operatorname{tang} \varrho^{\prime} \cos \psi & 1 & 1 & 1 \\
1 & \cos \mathfrak{e}_{11} & \cos \mathfrak{e}_{12} & \cos \mathfrak{e}_{13} \\
1 & \cos \mathfrak{e}_{21} & \cos e_{22} & \cos e_{23} \\
1 & \cos e_{31} & \cos \mathfrak{e}_{32} & \cos e_{33}
\end{array}\right|=0 .
$$

Wir leiten schliesslich aus (12.) und (17.) folgende Resultate her: aus (17.)

1) Lässt man die beiden Raumwinkel congruiren, so erhält man

$$
\text { (18.) }\left|\begin{array}{cccc}
\frac{1}{\cos ^{2} r^{\prime}} & 1 & 1 & 1 \\
1 & 1 & \cos \mathrm{e}_{12} & \cos \mathrm{e}_{13} \\
1 & \cos \mathrm{e}_{12} & 1 & \cos \mathrm{e}_{23} \\
1 & \cos \mathrm{e}_{13} & \cos \mathrm{e}_{23} & 1
\end{array}\right|=0
$$


Siebeck, Anwendung einer Determinante auf die Raumgeometrie.

und aus (12.)

$$
\left|\begin{array}{cccc}
1 & 1 & 1 & 1 \\
1 & 1 & \cos e_{12} & \cos e_{13} \\
1 & \cos e_{12} & 1 & \cos e_{23} \\
1 & \cos e_{13} & \cos e_{23} & 1
\end{array}\right|=-\sin ^{2} E_{1} E_{2} E_{3} \cdot \operatorname{tang}^{2} r^{\prime}
$$

2) Bezeichnen wir durch $D_{i k}$ und $D_{i k}^{\prime}$ die Winkel $E_{i} O E_{k}$ und $E_{i} O E_{k}$, so erhalten wir aus (12.) in Verbindung mit (19.) die der (9.) entsprechende Bedingungsgleichung für die Berührung der beiden Kegel

$(20).\left|\begin{array}{cccc}1 & 1 & 1 & 1 \\ 1 & \cos e_{11} & \cos e_{12} & \cos e_{13} \\ 1 & \cos e_{21} & \cos e_{22} & \cos e_{23} \\ 1 & \cos e_{31} & \cos e_{32} & \cos e_{33}\end{array}\right|-\left|\begin{array}{cccc}1 & 1 & 1 & 1 \\ 1 & 1 & \cos D_{12} & \cos D_{13} \\ 1 & \cos D_{12} & 1 & \cos D_{23} \\ 1 & \cos D_{13} & \cos D_{23} & 1\end{array}\right| \begin{array}{cccc}1 & 1 & 1 & 1 \\ 1 & 1 & \cos D_{12}^{\prime} & \cos D_{13}^{\prime} \\ 1 & \cos D_{12}^{\prime} & 1 & \cos D_{23}^{\prime} \\ 1 & \cos D_{13}^{\prime} & \cos D_{23}^{\prime} & 1\end{array} \mid=0$.

3) Die beiden Kegel schneiden sich rechtwinklig, wenn

$$
\left|\begin{array}{cccc}
1 & 1 & 1 & 1 \\
1 & \cos \mathfrak{e}_{11} & \cos \mathfrak{e}_{12} & \cos \mathfrak{e}_{13} \\
1 & \cos \mathfrak{e}_{21} & \cos \mathfrak{e}_{22} & \cos \mathfrak{e}_{23} \\
1 & \cos \mathfrak{e}_{31} & \cos \mathfrak{e}_{32} & \cos \mathfrak{e}_{33}
\end{array}\right|=0 .
$$

Insbesondere liegen die Geraden $O \mathrm{E}_{1}$ und $O \mathrm{E}_{2}$ mit der Achse des durch $O E_{1}$, $O E_{2}, O E_{3}$ gehenden Kegels in einer Ebene, wenn

$$
\text { (22.) }\left|\begin{array}{lll}
1 & \cos \mathfrak{e}_{11} & \cos \mathfrak{e}_{12} \\
1 & \cos \mathrm{e}_{21} & \cos \mathrm{e}_{22} \\
1 & \cos \mathrm{e}_{31} & \cos \mathrm{e}_{32}
\end{array}\right|=0 \text {. }
$$

Indem ich diese Betrachtungen schliesse, bemerke ich noch, dass ich, um nicht durch Weitschweifigkeit zu ermüden, die analogen Betrachtungen für die Ebene unterlassen habe. 